to the supervisory board. The concept of a health service corporation is not new. ${ }^{9}$ Indeed, the royal commission considered the idea and rejected it but with a caveat that it was a matter "that ministers should keep under review." Perhaps its time has come. Certainly, the proposal, with the implication that the Department of Health and Social Security would be much reduced in size and influence, is one that will appeal to this government. It is also a proposal that will provoke hostility from some members of parliament, who will see it as diminishing their influence; from the Civil Service, who will be losing jobs; and from some health service unions, who may see it as reducing the opportunities for lobbying or for holding up changes that they oppose. If it improves management, concentrates more resources on patient care, reduces interference from the DHSS, and improves industrial relations (the authors want personnel management strengthened) doctors should welcome the concept.

One recommendation that will be contentious but which is crucial to improving the speed and quality of decision making is that a health authority's general manager "would be the final decision taker for decisions normally delegated to the consensus team, especially where decisions cross professional boundaries or cause disagreement and delay at present." This modification of consensus management has already provoked hostile responses from the Royal College of Nursing, ${ }^{10}$ but the BMA has been more welcoming. The secretary, Dr John Havard, said last week that clinicians had become increasingly frustrated with the delays that accompanied even relatively unimportant decisions in the NHS. The association will study closely the detailed arrangements proposed in the Griffiths report and-in line with the policy of the representative body in 1980-it will challenge the concept of a non-medical chief executive.

Doctors will welcome the call for less of their time to be spent in committees and the proposals point the way to achieving this. More of their time can then be spent with patients - which, when all is said and done about management, is what the NHS is about. Fortunately, Mr Griffiths has not forgotten them. "It is central to the approach of management," his letter states, "to ascertain how well the service is being delivered . . . by obtaining the experience and perceptions of patients and the community ... respond directly to this information ... act on it . . . and monitor performance against it. . . " Coming from a senior executive of one of the most successful consumer oriented businesses in Britain, this advice should be heeded. The opinions of patients and the community deserve as careful a hearing as that of health professionals and managers. If this is seen to happen it should strengthen the NHS's ability to cope rationally with the unfair demands of the pressure groups that flourish round the fringes of the NHS.

1 Anonymous. Reorganisation à la carte. [Editorial.] $\mathrm{Br} \mathrm{Med} \mathcal{F} 1982 ; 284$ 769-70.

${ }^{2}$ Anonymous. Sir Derek Rayner to advise on NHS performance. $\mathrm{Br} \mathrm{Med} \mathrm{f}$ $1982 ; 284: 1208$.

${ }^{3}$ Department of Health and Social Security. Health services management performance indicators. HN(83)25. London: DHSS, 1983.

4 NHS Management Inquiry. Report. London: DHSS, 1983. (Griffiths report.)

${ }^{5}$ Russell W. Political parties fight to champion NHS. Br Med $\mathcal{f} 1983$; 287:1233.

${ }^{6}$ Hampton JR. The end of clinical freedom. [Editorial.] Br Med $\mathcal{F} 1983$; 287:1237-8.

${ }^{7}$ Royal Commission on the National Health Service. Report. Cmnd 7615. London: HMSO, 1979:298-331.

${ }^{8}$ Department of Health and Social Security. Management arrangements for the reorganised National Health Service. London: HMSO, 1972.

${ }^{9} \mathrm{~W}$ iseman J. A health corporation ? Br Med f 1967;ii:102-3.

10 Anonymous. Mixed reaction to inquiry findings. The Times 1983 Oct $26: 5$.

\section{Searching the literature}

Peter P Morgan, scientific editor of the Canadian Medical Association fournal, recently suggested" that "a literature search should be regarded as a 'method' just like a treatment or diagnostic procedure and should be described in the methods section of most papers." His intention was not that readers wishing to validate the work described in the paper should be able also to test the search procedure but that the editor might be reassured that the author had reviewed the published work adequately when the references cited were few or old or all to the author's own publications.

Surely this is a subversive suggestion? Firstly, it subverts the principle, often subverted, that journal articles are for the readers, who are collectively perfectly capable of judging the adequacy of the references. Secondly, it subverts the task of the referees. Thirdly, it subverts the function of citing references-which is to acknowledge relevant previous work but emphatically not to show that the author has looked up everything ever published on the subject. And, fourthly, it is subversive in conveying the idea that, after the problem and all possible solutions had been comprehensively analysed, this is the definitive answer. In fact, a moment's thought discloses many flaws: for example, there is nothing to say what the search produced, that the author heeded the results, or that it was carried out properly.

An author is usually at least as worried about the adequacy of his review of published work as the editor because he is afraid that the editor or the referees will know of publications that he has failed to turn up, however diligent his search has been. What is he to do? How much time before, during, and after doing the work can he afford to spend on searching the literature?

In practice the author has two sources of published knowledge: his own background knowledge and reading, however he stores and retrieves it, and what he looks up specifically for the work in hand. Background knowledge naturally has limitations, because interests change and future needs are difficult to predict. Nevertheless, a faint memory-from a journal club meeting, for example-can sometimes produce a lateral reference that no search would throw up.

When making specific searches most of us have until recently looked only retrospectively. We start from a recent article, textbook, monograph, or conference proceedings and work through the references cited and the references cited by them. Having defined the medical subject headings that cover the subject, we can also work retrospectively through the Index Medicus. For a fee we may order computer searches of various data bases in consultation with the librarian. Now, however, that the Science Citation Index of the Institute for Scientific Information is more generally available we can also search prospectively. Each year the staff there insert details (including all the references) of 600000 articles into a data base that now contains more than 900 million articles. This material is available in book form as well as in computer format for locating all the publications in which the initial reference has been cited since it was published. The drawback to all these search systems is that they retrieve far too many publications; extracting the ones that are relevant to the particular needs of one's paper may be very time consuming.

I believe that most authors use these methods to review the previous work in their subjects, and that Dr Morgan is worrying too much. Clearly in the vast array of publications no one can expect to find, read, or assess every relevant one, and nuggets 
will be missed. Each author must do his best, and the editor, advised by his referees, must judge whether that best is good enough. If the editor decides to publish his readers can judge and comment, either privately or in the correspondence column of the journal, if prior claims have been overlooked. They frequently do.

\section{W F WHIMSTER}

Reader and Honorary Consultant in Morbid Anatomy, King's College Hospital Medical School London SE5 8RX

1 Morgan PP. Covering and recovering the medical literature. Can Med Assoc 7 1983;128:1264.

\section{Alzheimer's disease: recent advances and future prospects}

Recent years have seen major advances in our understanding of the neurochemical basis of dementia of the Alzheimer type. Its distinctive neuropathological features include plaques, neurofibrillary tangles, and other changes, but three reports in $1976-8^{1-3}$ also described appreciable reductions in the activity of the enzyme choline acetyltransferase (which synthesises acetylcholine) in cerebral cortex from patients who had died of dementia of the Alzheimer type. These studies generated considerable interest in the cholinergic system in dementia: the currently influential theory that cholinergic dysfunction underlies the clinical features has recently been well reviewed by Coyle, Prince, and DeLong. ${ }^{4}$

Reductions in cortical choline acetyltransferase activity in postmortem or biopsy samples of brain occur in specific association with the classical neuropathological changes of Alzheimer's disease: they are not found consistently, for example, in multi-infarct dementia. Furthermore, the severity of the neuropathological changes is associated with the extent of the reductions in choline acetyltransferase and cognitive impairment. ${ }^{56}$ Alzheimer type neuropathological changes are most pronounced in the temporal cortex and hippocampus and so are the reductions in choline acetyltransferase activity; these changes may be responsible for the defects in new learning. Neocortical neuropathological lesions and reductions in choline acetyltransferase are more pronounced in patients with a younger age of onset, ${ }^{7}$ and these changes might underlie apraxias, aphasias, and agnosias.

Neuroanatomical studies suggest that the cortex does not contain intrinsic cholinergic cell bodies, but that it receives an extrinsic innervation of cholinergic nerve terminals originating from subcortical neurones of the substantia innominata, nucleus of the diagonal band of Broca, and septal nuclei." These areas are affected by Alzheimer type neuropathological lesions and, in one study, by neuronal fallout and reductions in cholinesterase staining which may indicate loss of cholinergic neurones. ${ }^{4}$ Rossor et $a l^{8}$ found substantial reductions of choline acetyltransferase activity in postmortem samples of the substantia innominata, whereas most other subcortical samples showed minor changes. If confirmed, these findings suggest that reductions in cortical choline acetyltransferase in
Alzheimer's disease result in part from degeneration of cholinergic neurones of the substantia innominata. Probably predegenerative changes also contribute to reduced choline acetyltransferase activity. Loss of cholinergic cell bodies may be due to retrograde degeneration or retrograde spread of some pathological agent from cortical lesions. There are indications that in cortical plaques the degenerate nerve terminals which cluster round the central amyloid core may be cholinergic ${ }^{4}$; if this proves to be the case the plaque may be the primary site of damage to the cholinergic system.

Most studies agree that there is no reduction in the numbers of muscarinic cholinergic receptors in Alzheimer's disease, ${ }^{4}$ suggesting that those intrinsic cortical cells which are postsynaptic to the dysfunctional or degenerating cholinergic afferents remain intact. This raises therapeutic possibilitiesfor the presynaptic deficit in cholinergic neurotransmission might be correctable with drugs which potentiate residual presynaptic function (for example, choline, acetylcholinesterase inhibitors) or which directly activate muscarinic receptors (for example, arecoline). Several trials of these agents have been reported but so far without any substantial improvements in tests of memory. ${ }^{4}$ It would be premature, however, to discard the idea that cholinergic dysfunction underlies some of the symptoms of dementia of the Alzheimer type. The drugs do not restore physiological spatial and temporal patterns of cholinergic neurotransmission; yet small but significant gains in test scores have been seen in some patients, particularly after physostigmine or arecoline. ${ }^{9}$ Furthermore, there is good evidence that in normal people and animals interference with cholinergic neurotransmission induced by drugs influences memory functions in appropriate directions. ${ }^{410}$

Coyle and colleagues ${ }^{4}$ describe Alzheimer's disease as a "disorder of cortical cholinergic innervation." While this identifies the main change and the part it may play in producing the disturbance of memory, the description perhaps shifts emphasis away from other neurochemical changes. Almost 20 years ago the tangle formation seen in Alzheimer's disease was known to have a predilection not only for cortical areas and the substantia innominata but also for brain stem nuclei such as the locus ceruleus and raphe nuclei ${ }^{11}$ - which give rise respectively to noradrenergic and serotoninergic innervation of the cortex. More recent studies have shown corresponding reductions in biochemical markers for noradrenergic and serotoninergic terminals in cortex from patients with Alzheimer's disease. ${ }^{412}$ Dopaminergic systems may also be affected.

The issue whether intrinsic cortical cells also degenerate in Alzheimer's disease is unresolved. Different populations of cortical cells are thought to contain $\gamma$-aminobutyric acid and the peptides cholecystokinin, vasoactive intestinal polypeptide, and somatostatin. Reductions in $\gamma$-aminobutyric acid and somatostatin concentrations have been described in cortical samples affected by Alzheimer's disease whereas vasoactive intestinal polypeptide and cholecystokinin are unaffected. ${ }^{4} 713$ This may indicate degeneration of certain cortical cells but might also be a transsynaptic effect of the degeneration of afferent projections.

The ubiquity and the parts played by deficits in noncholinergic systems in Alzheimer's disease remain to be established. Monoamines are concerned with attentional, motivational, sleep, and learning processes-and in the pathogenesis of affective and other psychoses-and they seem likely to contribute to the pattern of symptoms in many people with Alzheimer's disease.

Clearly further correlative studies are needed to assess 